\title{
A Miniature Droplet Reactor Built on Nanoparticle-Derived Superhydrophobic Pedestals
}

\author{
Bin Su, Shutao Wang, Yanlin Song, and Lei Jiang ( $\square)$ \\ Beijing National Laboratory for Molecular Science (BNLMS), Institute of Chemistry, Chinese Academy of Sciences, Beijing 100190, China \\ Received: 18 October 2010 / Revised: 6 November 2010 / Accepted: 10 November 2010 \\ (C) Tsinghua University Press and Springer-Verlag Berlin Heidelberg 2010
}

\begin{abstract}
The capability to design and modulate materials, shapes, heat transfer, and mass mixing during the process of developing chemical reactors has allowed researchers to explore millions of chemical reactions and assays. However, despite the advantages in engineering array-based microreactors or microfluidic systems, the wetting attachment between solutions of reagents/products and the glass or polymer substrates of containers leads to difficulties in collecting products effectively and preventing channel blockage. Herein we present a miniature droplet reactor which takes advantage of the anti-wetting and low-adhesive properties of nanoparticle-derived superhydrophobic pedestals, allowing aqueous droplets to be manipulated freely but also providing a confined environment for performing a series of aqueous phase chemical reactions on a small scale. Gas- or precipitateforming reactions can also be performed inside this miniature reactor. Most importantly, reaction products in liquid, solid or gaseous states can be collected effectively, which allows the harvesting of valuable products formed in limited amounts. Such a miniature reactor built on superhydrophobic pedestals provides a new way of performing common chemical reactions and may open the door to the design of next-generation microreaction systems.
\end{abstract}

\section{KEYWORDS}

Superhydrophobic, reactor, droplet, hybrid materials, nanomaterials

\section{Introduction}

Billions of chemical reactions have been performed in test tubes, flasks or other glass containers since the establishment of modern chemistry by Lavoisier in 1775 $[1,2]$. Over the past decade, microreactor technology, such as microemulsions [3], array-based microreactors [4] or microfluidic systems [5-7], has attracted great interest since it enables the miniaturization of reactions by compartmentalizing reactions in droplets of nanoliter to microliter volumes. Notably, droplet-based microfluidic systems, in which reactions are performed by mixing aqueous reagent droplets in oil-filled microchannels, have shown promising applications in enzymatic kinetics, protein crystallization, and other biochemical reactions [8]. Since weak van der Waals forces or stronger hydrogen bonds generally exist when water molecules are close to the molecules/atoms on solid surfaces [9-11], problems of adhesion between the aqueous liquid and container walls usually occur in such reactors. As a result, due to the wetting nature of the contact, liquids with reaction products tend to remain at the bottom of containers or micro-channels, which can simultaneously decrease product yield, clog reactors, and result in contamination. In order to overcome the adhesion problem and collect reaction

Address correspondence to jianglei@iccas.ac.cn 
products effectively, bio-inspired superhydrophobic materials [12-18] might be a good candidate for a reactor surface owing to their excellent anti-wetting and low-adhesive properties [19, 20].

Herein we demonstrate a miniature droplet reactor based on nanoparticle-derived superhydrophobic pedestals, which provides a confined space with the capability to perform a series of aqueous phase chemical reactions. A facile "glue and nanoparticles" method is employed to form superhydrophobic coatings on glass or metallic pedestals. Due to the well known water-repellent and anti-adhesion properties of superhydrophobic materials, water droplets can be manipulated by a superhydrophobic clip. Then, the coalescence of droplets containing different reagents leads to chemical reactions inside the final coalesced droplet. Different types of aqueous phase inorganic/ organic chemical reactions have been performed inside the miniature reactor to show its wide applicability. Gas- or precipitate-forming reactions have also been performed inside this miniature reactor. Reaction products in liquid, solid or gaseous states can be collected effectively, which allows the harvesting of valuable reaction products formed in limited amounts. Our results suggest that such a miniature reactor built on superhydrophobic pedestals provides a new way to carry out common chemical reactions and new ideas for the design of next-generation microreaction devices.

\section{Experimental}

\subsection{Fabrication of the superhydrophobic pedestals}

$1 \mathrm{~g}$ of polystyrene granules $\left(M_{\mathrm{W}} 192,000 \mathrm{~g} / \mathrm{mol}\right.$, Aldrich), $0.8 \mathrm{~g}$ of hydrophobic fumed silica nanoparticles (Aerosil R202, average particle size $14 \mathrm{~nm}$, Evonik Degussa Co.) and $30 \mathrm{~mL}$ of chloroform were mixed and stirred for $30 \mathrm{~min}$ in a closed bottle. Precleaned glass plates were dipped into this sticky solution, held for $1 \mathrm{~min}$, and pulled up at a speed of ca. $0.5 \mathrm{~cm} / \mathrm{s}$. Owing to the rapid evaporation of solvent, the semi-transparent membrane quickly transformed into a white coating with extremely high waterrepellency. The coated plates were dried at room temperature and then heated at $80{ }^{\circ} \mathrm{C}$ for $1 \mathrm{~h}$, yielding a hard coating on the surface. For fabrication of superhydrophobic clips, the volume of chloroform was decreased to $15-18 \mathrm{~mL}$ in order to make the composite solution sufficiently sticky, and the resulting mixture was coated on aluminium clips with all the other details being the same.

\subsection{Characterization}

The structures of the composite coatings on substrates were examined using a scanning electron microscope (SEM, JEOL, JSM-6700F, Japan) with an accelerating voltage of $3.0 \mathrm{kV}$ and equipped with an energy dispersive X-ray spectroscopy (EDS) detector. Static water contact angles (CA) were measured on a DataPhysics OCA20 contact angle system at ambient temperature. The average CA was obtained by measuring more than five different positions on the same sample. The adhesive force was measured by removing a $10 \mu \mathrm{L}$ microdroplet from a coated layer using a high-sensitivity microelectromechanical balance system with a resolution of $10 \mu \mathrm{g}$ (DataPhysics DCAT 11, Germany) and a charge-coupled device (CCD) camera system at ambient temperature. When performing chemical reactions on superhydrophobic pedestals, droplets were colored by various chemical reagents for easy observation. Different solutions were prepared in volumetric flasks and slowly dropped onto the superhydrophobic plates. The volume of water droplets was controlled by a Finnpipette (Thermo Fisher Scientific Oy, Finland) which can release droplets on a controllable microliter level. The videos or photographs were recorded using a commercial 7.2-megapixel digital camera (Sony, DSC-W120, Japan).

\section{Results and discussion}

\subsection{Droplets on superhydrophobic pedestals serving as microscale "containers"}

Research on superhydrophobic materials to date has mainly focused on techniques for preparing rough solid surfaces in an inexpensive and simple manner [21]. However, less attention has been paid to droplets on superhydrophobic surfaces. In this study, a superhydrophobic plate was fabricated through a "glue and nanoparticles" method (details of the structure 
and superhydrophobicity of the composite coatings can be found in Fig. S-1 in the Electronic Supplementary Material (ESM)). Then, a ca. $20 \mu \mathrm{L}$ droplet, in which trapped a Caridina serrata type shrimp, was carefully placed on the superhydrophobic plate which was kept horizontal. Owing to the excellent water-repellent and low-adhesive properties of the coated plate, the droplet formed a perfect spherical shape with minimum surface free energy (Fig. 1(a)). Interestingly, unlike its streamlined appearance in a Petri dish filled with water (Fig. S-2(a) in the ESM), the shrimp became significantly bent. It is believed that such a posture of the shrimp results from the existence of an invisible hydrogen-bonding net around the droplet [22]. The conformation of water molecules closest to the interface has been shown theoretically to depend strongly on the wetting properties of the underlying solid surface [23]. On the superhydrophobic pedestals, interfacial water molecules become arrayed in a regular structure in order to maximize their hydrogen-bonding partners and thereby form a compact hydrogenbonding net (Fig. 1(b)), resulting in higher density than that of the adjacent layer [24]. Due to the "hard wall" effect of the hydrogen-bonding net [25], bulk water inside droplets is isolated from the waterrepellent surface and a microscale "container" is thus formed around each droplet on the superhydrophobic pedestals.

To demonstrate the wide applicability of such a "container" for holding and confining various guests, we placed droplets with different concentrations of reagents or $\mathrm{pH}$ values onto the anti-wetting plates. When the concentration of sodium chloride was varied from 0.001 to $5 \mathrm{~mol} / \mathrm{L}$, the superhydrophobic pedestals used in this study showed great repellence for all droplets (Fig. S-2(b) in the ESM) and similar effects were observed when the $\mathrm{pH}$ was varied from 1 to 13 (Fig. S-2(c) in the ESM); these results indicate the excellent ability of such a "container" to retain different chemical reagents. Therefore, each droplet on the superhydrophobic pedestals can serve as a microscale "container" for storing various chemical reagents.

\subsection{On-demand manipulation of droplets on superhydrophobic pedestals}

Furthermore, the "containers" on superhydrophobic pedestals can be manipulated at will as shown in Fig. 2. We built a superhydrophobic system consisting of highly hydrophobic clips and plates prepared using the same coating method. Owing to the excellent water-repellent properties of the coated plate, a $20 \mu \mathrm{L}$ water droplet displayed spherical shape (Fig. 2(a)). Due to the existence of the hydrogen-bonding net around it, the droplet could be lifted intact from the superhydrophobic surface by closing the tips of the superhydrophobic clip (Figs. 2(b) and 2(c)). Through this "mechanical arm" - like clip motion, by closing and opening the tips, the droplet could be placed at will on the superhydrophobic plate (Fig. 2(d)). Besides

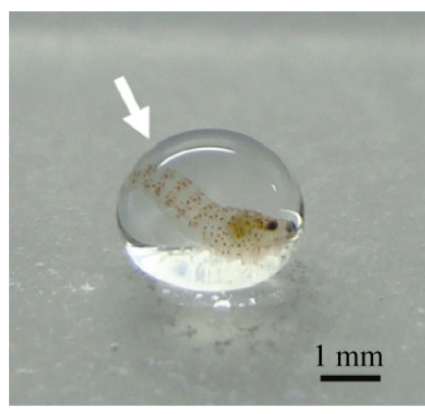

(a)

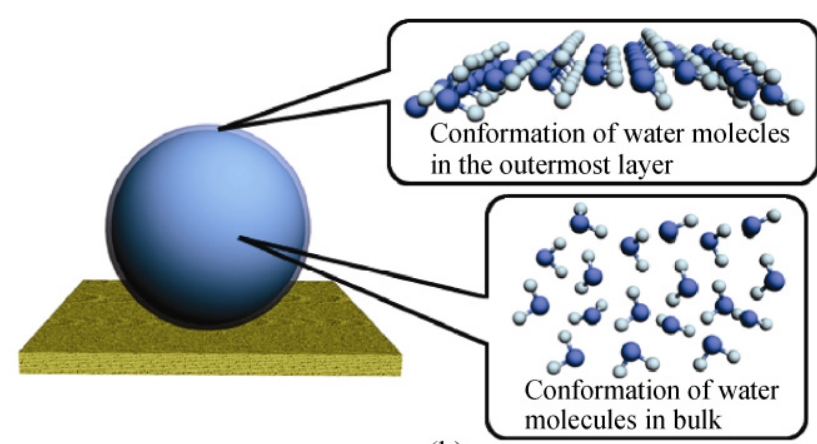

(b)

Figure 1 (a) Optical photograph of a $c a .20 \mu \mathrm{L}$ droplet, in which a Caridina serrata type shrimp was trapped, placed on the superhydrophobic pedestal. (b) Schematic illustration of a droplet on a superhydrophobic plate, showing the invisible hydrogen-bonding net around the droplet consisting of a regular-array of water molecules. The conformation of water molecules closest to the water/solid surface and water/atmosphere interfaces is different from that in the bulk. Interfacial molecules form regular structure in order to maximize their hydrogen-bonding partners and thereby form a compact hydrogen-bonding net 
transferring a single water droplet, sequencing different droplets (Fig. S-3 in the ESM), and collecting small droplets to form a larger one (Fig. S-4 in the ESM) can also be achieved by such manipulation. Here we should point out, although such on-demand manipulation of $20 \mu \mathrm{L}$ droplets can also be obtained with a normal pipette, the adhesion between the droplets and pipette inner wall will inevitably result in loss of droplets. By virtue of the water-repellent and low-adhesive properties of the superhydrophobic pedestals, liquid droplets can be held and moved at will, and such manipulation facilitates the harvesting of valuable materials formed in limited amounts. The process of directly manipulating a water droplet can be performed without the aid of functional additives [26-28] such as Janus silica particles or $\mathrm{Fe}_{3} \mathrm{O}_{4}$ nanoparticles, and/or applied force fields [29-32] such as magnetic, electrical or optical. Since no additives are required, on-demand droplet movements are possible without any possibility of contamination.

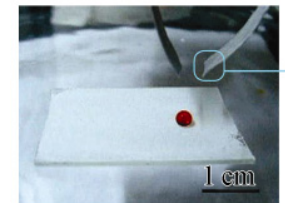

(a)

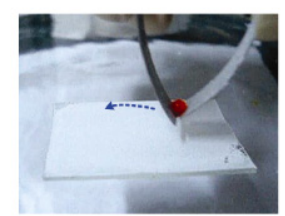

(c)
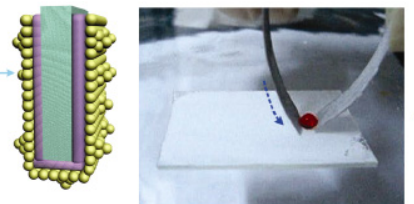

(b)
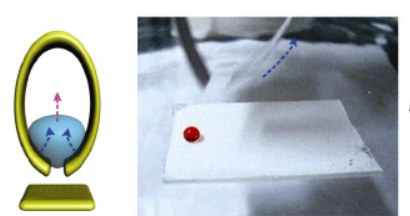

(d)
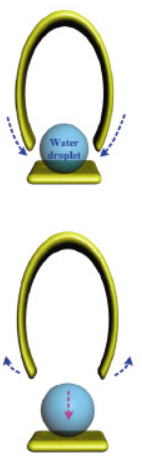

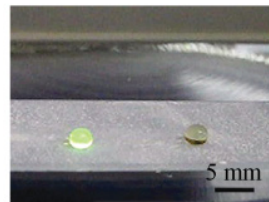

(a)

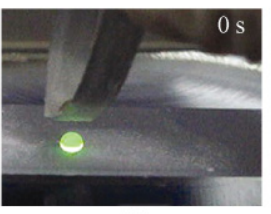

(b)

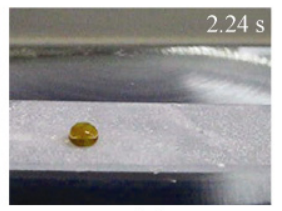

(c)

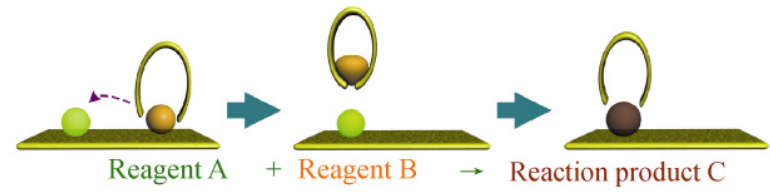

(d)

Figure 3 Photographs of each stage of the fluorescence quenching reaction. (a) A droplet of $0.05 \mathrm{~mol} / \mathrm{L}$ calcein solution was carefully placed on the anti-wetting plate, forming a spherical shape with yellowish-green fluorescence under irradiation by a $16 \mathrm{~W}$ ultraviolet lamp at distance of ca. $0.1 \mathrm{~m}$. (b) A pale-yellow droplet of $0.05 \mathrm{~mol} / \mathrm{L} \mathrm{FeCl}_{3}$ solution was picked by a superhydrophobic clip and dropped onto the fluorescent droplet. (c) The coalesced droplet quickly changed color from fluorescent yellowishgreen to gray-brown, indicative of attachment of $\mathrm{Fe}^{3+}$ ions to calcein molecules. (d) Schematic illustration of the process for performing chemical reactions on superhydrophobic pedestals 
Therefore, a new concept of manipulation of microscopic chemical reactions on superhydrophobic pedestals can be described as follows (Fig. 3(d)): One water droplet (containing reagent $\mathrm{A}$ ) on a superhydrophobic pedestal was coalesced with another droplet (containing reagent $\mathrm{B}$ ) by means of a superhydrophobic clip, resulting in a final droplet (a microreactor containing product $\mathrm{C}$ ). To further demonstrate how coalescence of droplets containing different reagents can lead to chemical reactions inside the final miniature droplet reactor, we carried out six typical inorganic reactions utilizing the miniature reactors on superhydrophobic pedestals including a decomposition reaction (Fig. S-5 in the ESM), neutralization reactions (Figs. 4(e)-4(g) and Fig. S-6 in the ESM), a displacement reaction (Fig. S-7 in the ESM), a gas-forming metathesis reaction (Fig. S-8 in the ESM), and a precipitate-forming metathesis reaction (Figs. 4(a)-4(c)). Here we should point out that only reactions of watermiscible reagents can be performed in this miniature reactor since the superhydrophobic pedestals in this study are unable to bear organic solvents. However, several aqueous phase organic reactions, such as addition reactions, substitution reactions, oxidation reactions (Fig. S-9 in the ESM) and a typical organic color-forming reaction between phenol and $\mathrm{Fe}^{3+}$ (Fig. S-10 in the ESM) could be realized by means of the anti-wetting clips and plates. Therefore, this miniature reactor showed wide adaptability for aqueous phase reactions.

In contrast to the problems of product loss occurring in conventional microreactor systems due to the

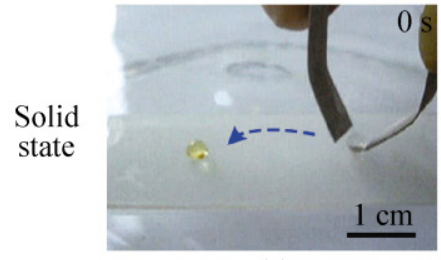

(a)

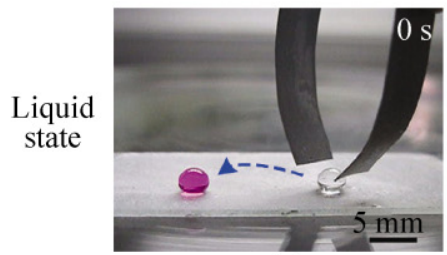

(e)

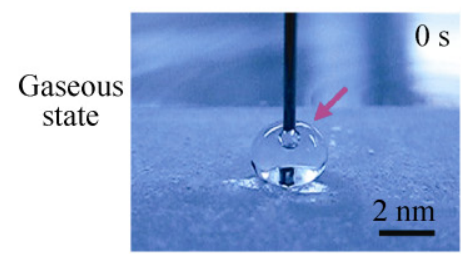

(i)

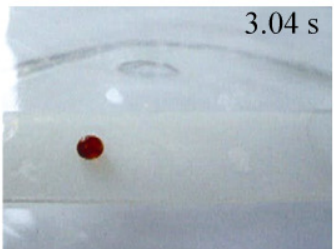

(b)

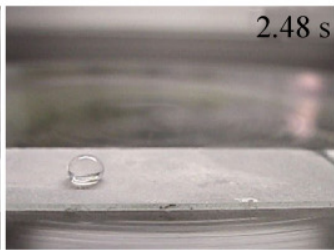

(f)

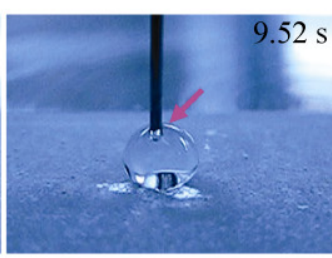

(j)

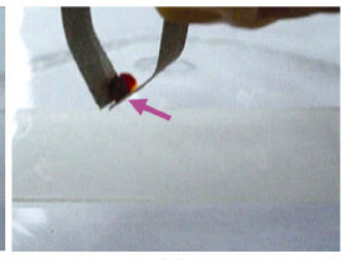

(c)

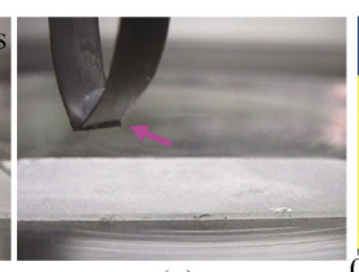

(g)

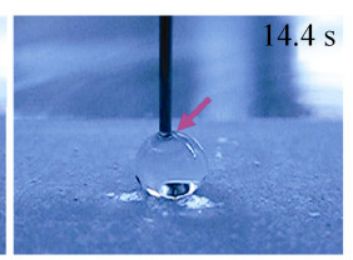

(k)

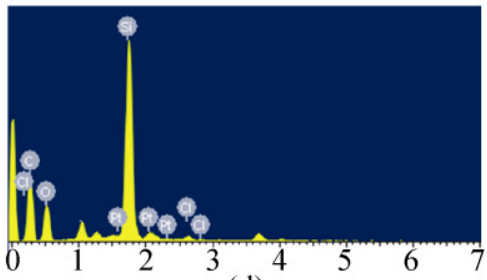

(d)

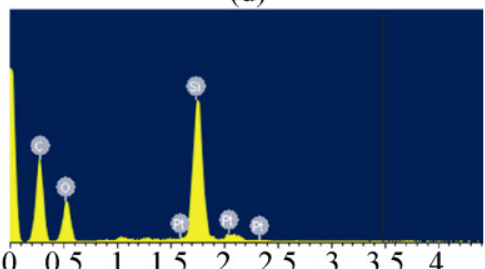

(h)

Figure 4 Photographs of the stages of different reactions. (a)-(c) Collection of a solid product. A precipitate-forming metathesis reaction was performed by combining a yellow droplet of $1 \mathrm{~mol} / \mathrm{L} \mathrm{FeCl}_{3}$ and a colorless droplet of $1 \mathrm{~mol} / \mathrm{L} \mathrm{NaOH}$ solution. A red-brown sediment of $\mathrm{Fe}(\mathrm{OH})_{3}$ was generated inside the coalesced droplet and could be collected completely following the removal of the final droplet microreactor with a superhydrophobic clip, leaving a clean superhydrophobic surface. (d) EDS analysis of the superhydrophobic surface after the precipitate-forming metathesis reaction and removal of the coalesced droplet showing the absence of any iron-containing material on the surface. (e)-(g) Collection of a product dissolved in water. A neutralization reaction was performed by combining a pink droplet of $1 \mathrm{~mol} / \mathrm{L} \mathrm{NaOH}$ containing $0.05 \mathrm{~mol} / \mathrm{L}$ phenolphthalein and a colorless droplet of $1 \mathrm{~mol}^{-} \mathrm{L} \mathrm{H}_{2} \mathrm{SO}_{4}$ solution. The coalesced droplet quickly changed from pink to colorless and could be collected by a superhydrophobic clip. (h) EDS analysis of the superhydrophobic surface after the neutralization reaction and removal of the coalesced droplet. (i)-(k) Collection of a product in the gaseous state. $\mathrm{A} \mathrm{CO}_{2}$ bubble was generated by the coalescence of a droplet of $0.4 \mathrm{~mol} / \mathrm{L} \mathrm{NaHCO}$ solution and another droplet of $2.5 \mathrm{~mol} / \mathrm{L}$ hydrochloric acid (the amount of reagents should be controlled carefully to form only one bubble). The spherical bubble confined inside the droplet could be removed by a syringe needle 
adhesion between the aqueous liquid and the container walls [9-11], the anti-adhesive property of the superhydrophobic pedestals enabled the products from this miniature reactor to be harvested effectively. To demonstrate the collection of a solid product, a typical precipitate-forming metathesis reaction was performed. A colorless droplet of $1 \mathrm{~mol} / \mathrm{L} \mathrm{NaOH}$ solution was coalesced into a yellow droplet of $1 \mathrm{~mol} / \mathrm{L}$ $\mathrm{FeCl}_{3}$ solution by a superhydrophobic clip (Fig. 4(a)). After 3.04 seconds, a red-brown $\mathrm{Fe}(\mathrm{OH})_{3}$ sediment was generated and confined inside the coalesced droplet by the compact hydrogen-bonding net (Fig. 4(b)). The insoluble solid product could be collected completely by removal of the final droplet microreactor (Fig. 4(c)); this was confirmed by the absence of any signals from iron-containing materials when the superhydrophobic surface was analyzed by EDS (Fig. 4(d)). Reaction products in the liquid state or dissolved in water droplets can also be collected. A colorless droplet of $1 \mathrm{~mol} / \mathrm{L}$ sulfuric acid was picked up by a superhydrophobic clip and dropped onto a pink droplet of $1 \mathrm{~mol} / \mathrm{L} \mathrm{NaOH}$ solution containing $0.05 \mathrm{~mol} / \mathrm{L}$ phenolphthalein (Fig. 4(e)). The coalesced droplet quickly changed from pink to colorless in 2.48 seconds, which showed that a neutralization reaction was occurring inside the miniature reactor (Fig. 4(f)). The droplet containing the soluble product could be picked up and collected (Fig. 4(g)), as shown by the absence of any peaks from sulfur-containing compounds in the EDS analysis (Fig. 4(h)) of the superhydrophobic surface after removal of the coalesced droplet. Finally, if the product was in gaseous state such as a $\mathrm{CO}_{2}$ bubble generated by the coalescence of a droplet of $0.4 \mathrm{~mol} / \mathrm{L} \mathrm{NaHCO}_{3}$ solution and another droplet of $2.5 \mathrm{~mol} / \mathrm{L}$ hydrochloric acid (the amount of reagents should be controlled carefully to form only one bubble), the spherical bubble confined inside the droplet can be removed through a syringe needle in 14.4 seconds (Figs. 4(i)-4(k)). In summary, such a miniature reactor built on superhydrophobic pedestals offers effective ways of collecting reaction products in solid, liquid, and gaseous states due to the confining effect of the hydrogen-bonding net around the droplets [25].

Generally, microreactors such as microfluidic systems do not tolerate the formation of particulates because the microchannels tend to become clogged. Clogging has been identified by a number of researchers as the biggest hurdle to overcome if microreactors are to be widely accepted. Similarly, if gas bubbles are generated in aqueous droplet-based microreactors or diffuse inside the carrier fluid through the microchannels, these might also clog the microreactors and gradually shorten the residence time of reagents. In our case, the chemical reactions occur inside coalesced miniature reactors which are free of solid containers or microchannels. Therefore, precipitation and gas forming reactions can be performed successfully without any problems due to clogging. Such a miniature reactor built on superhydrophobic pedestals therefore does not suffer from the drawbacks of conventional dropletbased microfluidic systems.

Other factors need to be taken into consideration when constructing a new miniature reactor system, however: Firstly, the durability of the superhydrophobic coating was investigated. The "lifting-placing a droplet" cycles were repeated several times and good water-repellent properties remained on both clips and plates. Importantly, by virtue of the facile nature of the "glue and nanoparticles" method, the old coatings can be replaced quickly (with a fabrication time of just $2 \mathrm{~h}$ ) by a new layer if some defects appeared on the surfaces. Secondly, the volume of the water droplet was also varied. A droplet as small as $3 \mu \mathrm{L}$ can be manipulated by our special clips and larger volumes were even easier to pick up. Thirdly, problems associated with evaporation of water from droplets during slow reactions can be overcome by enlarging the volume of the droplet or increasing environmental humidity, allowing the lifetime of the miniature reactors to be prolonged. We are currently extending our studies to include reactions inside aqueous droplets in oil or organic droplets in water as a way of reducing evaporation and dealing with water-immiscible reagents, respectively.

\section{Conclusions}

A miniature droplet reactor on superhydrophobic pedestals has been successfully fabricated. The compact hydrogen-bonding net around the droplets played a key role in forming miniature reactors, where microparticles, precipitates or bubbles can be held and 
confined inside. The hydrogen-bonding net serves as an invisible "container", offering the capability to perform a series of aqueous phase inorganic/organic chemical reactions inside the miniature reactor. Most importantly, this miniature reactor allows solid, liquid, and gaseous products to be easily collected due to the "hard wall" effect of hydrogen-bonding net, allowing valuable products to harvested even when formed in limited amounts. This confined miniature reactor built on superhydrophobic pedestals should have numerous promising applications in microreactors, microcollectors, microsensors, and other fields.

\section{Acknowledgements}

The authors are grateful for a grant from the Major State Basic Research Development Program (No. 2007CB936403), the National Natural Science Foundation of China (No. 20571077), and the China Postdoctoral Science Foundation (No. 20100470557).

Electronic Supplementary Material: Supplementary material (further details of structural information and superhydrophobicity of superhydrophobic pedestals, manipulation of droplets, and various chemical reactions performed on superhydrophobic pedestals) is available in the online version of this article at http://dx.doi.org/10.1007/s12274-010-0078-5.

\section{References}

[1] Crosland, M. Lavoisier: Chemist, biologist, economistPoirier, JP. Nature 1997, 387, 250-251.

[2] Chertok, L.; Stengers, I. Therapy and the ideal of chemistry. Nature 1987, 329, 768.

[3] Lopez-Quintela, M. A.; Tojo, C.; Blanco, M. C.; Rio, L. G.; Leis, J. R. Microemulsion dynamics and reactions in microemulsions. Curr. Opin. Colloid. In. 2004, 9, 264-278.

[4] Gross, G. A.; Wurziger, H.; Schlingloff, G.; Schober, A. Microreactor array assembly, designed for diversity oriented synthesis using a multiple core structure library on solid support. Qsar. Comb. Sci. 2006, 25, 1055-1062.

[5] deMello, A. J. Control and detection of chemical reactions in microfluidic systems. Nature 2006, 442, 394-402.

[6] Kobayashi, J.; Mori, Y.; Okamoto, K.; Akiyama, R.; Ueno, M.; Kitamori, T.; Kobayashi, S. A microfluidic device for conducting gas-liquid-solid hydrogenation reactions. Science
2004, 304, 1305-1308.

[7] Haswell, S. J. Chemical technology-All together now. Nature 2006, 441, 705.

[8] Song, H.; Chen, D. L.; Ismagilov, R. F. Reactions in droplets in microflulidic channels. Angew. Chem. Int. Edit. 2006, 45, 7336-7356.

[9] Adams, J. B.; Hector, L. G.; Siegel, D. J.; Yu, H. L.; Zhong, J. Adhesion, lubrication, and wear on the atomic scale. Surf. Interface. Anal. 2001, 31, 619-626.

[10] Cognard, J. Some recent progress in adhesion technology and science. C. R. Chim. 2006, 9, 13-24.

[11] Marshall, S. J.; Bayne, S. C.; Baier, R.; Tomsia, A. P.; Marshall, G. W. A review of adhesion science. Dent. Mater. 2010, 26, E11-E16.

[12] Blossey, R. Self-cleaning surfaces-virtual realities. Nat. Mater. 2003, 2, 301-306.

[13] Chaudhury, M. K.; Whitesides, G. M. How to make water run uphill. Science 1992, 256, 1539-1541.

[14] Gao, X. F.; Jiang, L. Water-repellent legs of water striders. Nature 2004, 432, 36.

[15] Li, X. M.; Reinhoudt, D.; Crego-Calama, M. What do we need for a superhydrophobic surface? A review on the recent progress in the preparation of superhydrophobic surfaces. Chem. Soc. Rev. 2007, 36, 1350-1368.

[16] Zheng, Y. M.; Bai, H.; Huang, Z. B.; Tian, X. L.; Nie, F. Q.; Zhao, Y.; Zhai, J.; Jiang, L. Directional water collection on wetted spider silk. Nature 2010, 463, 640-643.

[17] Daniel, S.; Chaudhury, M. K.; Chen, J. C. Past drop movements resulting from the phase change on a gradient surface. Science 2001, 291, 633-636.

[18] Yang, J. T.; Yang, Z. H.; Chen, C. Y.; Yao, D. J. Conversion of surface energy and manipulation of a single droplet across micropatterned surfaces. Langmuir 2008, 24, 9889-9897.

[19] Sun, T. L.; Feng, L.; Gao, X. F.; Jiang, L. Bioinspired surfaces with special wettability. Accounts. Chem. Res. 2005, 38, 644-652.

[20] Liu, M. J.; Zheng, Y. M.; Zhai, J.; Jiang, L. Bioinspired super-antiwetting interfaces with special liquid-solid adhesion. Accounts. Chem. Res. 2009, 43, 368-377.

[21] Roach, P.; Shirtcliffe, N. J.; Newton, M. I. Progess in superhydrophobic surface development. Soft Matter. 2008, 4, 224-240.

[22] van Oss, C. J.; Giese, R. F. Role of the properties and structure of liquid water in colloidal and interfacial systems. J. Disper. Sci. Technol. 2004, 25, 631-655.

[23] Trudeau, T. G.; Jena, K. C.; Hore, D. K. Water structure at solid surfaces of varying hydrophobicity. J. Phys. Chem. C. 2009, 113, 20002-20008.

[24] Matta, C. F.; Hernandez-Trujillo, J.; Tang, T. H.; Bader, R. 
F. W. Hydrogen-hydrogen bonding: A stabilizing interaction in molecules and crystals. Chem. Eur. J. 2003, 9, 1940-1951.

[25] Abraham, F. F. The interfacial density profile of a LennardJones fluid in contact with a (100) Lennard-Jones wall and its relationship to idealized fluid/wall systems: A Monte Carlo simulation. J. Chem. Phys. 1978, 68, 3713-3716.

[26] Kim, S. H.; Lee, S. Y.; Yang, S. M. Janus microspheres for a highly flexible and impregnable water-repelling interface. Angew. Chem. Int. Edit. 2010, 49, 2535-2538.

[27] Hong, X.; Gao, X. F.; Jiang, L. Application of superhydrophobic surface with high adhesive force in no lost transport of superparamagnetic microdroplet. J. Am. Chem. Soc. 2007, 129, 1478-1479.

[28] Zhao, Y.; Fang, J.; Wang, H. X.; Wang, X. G.; Lin, T. Magnetic liquid marbles: Manipulation of liquid droplets using highly hydrophobic $\mathrm{Fe}_{3} \mathrm{O}_{4}$ nanoparticles. Adv. Mater. 2010, 22, 707-710.

[29] Zhang, K.; Liang, Q. L.; Ma, S.; Mu, X. A.; Hu, P.; Wang, Y. M.; Luo, G. A. On-chip manipulation of continuous picoliter-volume superparamagnetic droplets using a magnetic force. Lab. Chip. 2009, 9, 2992-2999.

[30] Velev, O. D.; Prevo, B. G.; Bhatt, K. H. On-chip manipulation of free droplets. Nature 2003, 426, 515-516.

[31] Hunt, T. P.; Issadore, D.; Westervelt, R. M. Integrated circuit/microfluidic chip to programmably trap and move cells and droplets with dielectrophoresis. Lab. Chip 2008, 8, 81-87.

[32] Ichimura, K.; Oh, S. K.; Nakagawa, M. Light-driven motion of liquids on a photoresponsive surface. Science 2000, 288, 1624-1626. 